\section{Gastric giveaways}

NKX2-1 is a crucial regulator of pulmonary differentiation that is often overexpressed in lung adenocarcinomas, and its loss correlates with a poor prognosis. Published data from Tyler Jacks and colleagues have shown that NKX2-1 can restrain the capacity of oncogenic KRAS-driven lung cancers to evolve as a poorly differentiated, more aggressive disease. Jacks and colleagues now have a potential mechanism to explain this result.

The authors generated mice in which an allele of oncogenic $\operatorname{Kras}\left(\operatorname{Kras}^{G 12 D}\right)$ and both alleles of Nkx2-1 could be activated and deleted, respectively, from the lungs. Tumours in which Nkx2-1 was deleted had a strikingly different morphology (glandular and mucinous) from those expressing NKX2-1 (papillary-like and nonmucinous). Tumour burden was also greater in mice in which $N k \times 2-1$ was deleted owing to a greater number of initial lesions rather than to increased rates of proliferation. However, deletion of $N k \times 2-1$ in established tumours (3-7 months after $\operatorname{Kras}^{G 12 D}$ expression) led to a substantial change in the rate of proliferation; this increased tumour burden, and tumour cells in which Nkx2-1 was deleted progressively adopted a mucinous phenotype. These findings indicate that loss of Nkx2-1 both promotes tumour initiation and progression, and alters lung tumour cell fate.

Why does loss of Nkx2-1 result in a mucinous, glandular phenotype? Comparison of mRNA expression levels from the different tumour models described above, along with MetaCore pathway analyses, indicated that Nkx2-1 loss results in cells adopting an mRNA profile that is closest to that of the stomach epithelium. The HNF4a transcription factor that regulates gastrointestinal differentiation was expressed in all Nkx2-1-deleted tumours. Moreover, in 37 human lung adenocarcinoma samples, two stomach epitheliumrestricted proteins (GKN1 and CTSE) were predominately expressed in the NKX2-1-negative tumours. So, does NKX2-1 repress the expression of these stomach epithelium-specific genes? The authors could not find evidence that NKX2-1 functions as a direct repressor of such genes; however, many of the genes that had an increased level of expression as a result of NKX2-1 loss were bound by Forkhead box A1 (FOXA1) and FOXA2 transcription factors. Careful genetic and epigenetic analyses indicated that NKX2-1 recruits FOXA1 and FOXA2 to a pulmonary-specific set of genes and promotes their expression. However, when NKX2-1 expression is absent, FOXA1 and FOXA2 bind to new sites in the genome, many of which are near genes that are normally expressed in the gastrointestinal tract.

Further conditional gene deletion studies indicated that HNF4a is partly responsible for the gastric phenotype that is evident in Nkx2-1deleted lung tumours, but that deletion of both Hnf4a and Nkx2-1 did not result in any morphological changes: these tumours remained mucinous and well differentiated, and grew poorly. This is an interesting finding because loss of Hnf4a and Nkx2-1 leads to the partial derepression of $\mathrm{Hmg} 2 \mathrm{a}$, an embryonal transcription factor that is associated with the development of poorly differentiated, poor-prognosis

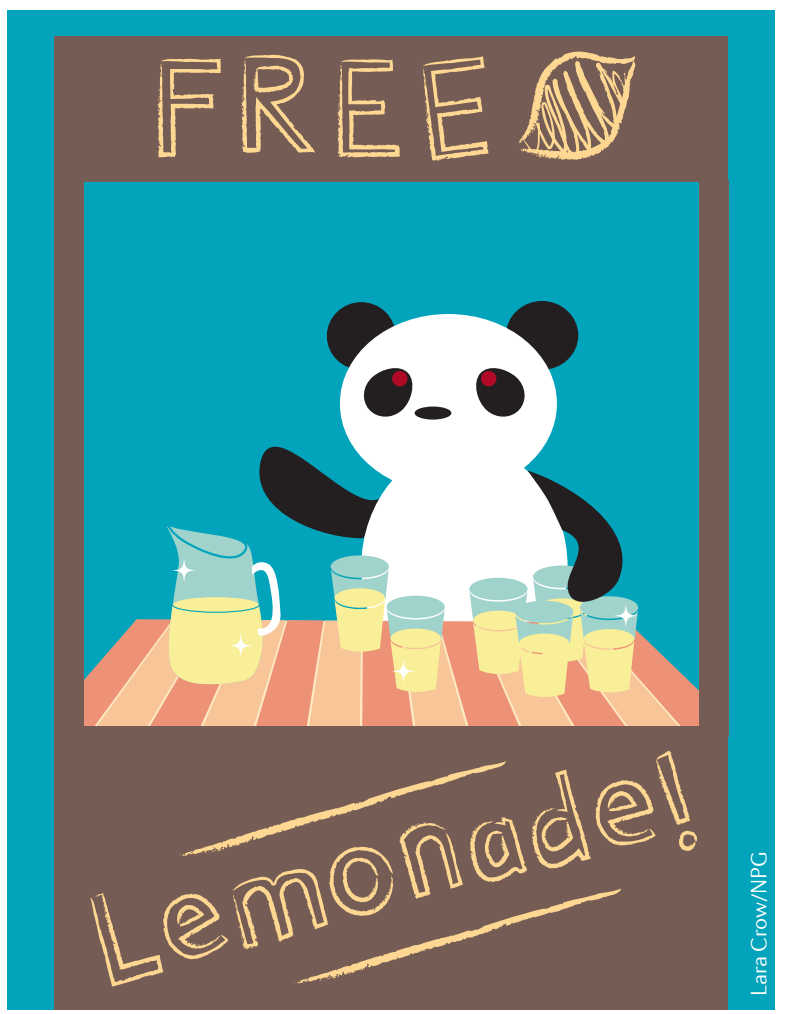

lung adenocarcinomas that have lost expression of NKX2-1. Thus, although HNF4a can repress Hmg2a, the loss of both Nkx2-1 and Hnf4a does not seem to convey a selective advantage.

These results indicate that NKX2-1 drives a pulmonary-specific differentiation programme and that this represses a default gastrointestinal differentiation pathway that involves HNF4a. However, even if both of these cell fate transcription factors are lost in lung epithelial cells, this does not automatically result in a more primitive cell type, suggesting that other programmes need to be overcome for lung tumour cells to adopt a truly dedifferentiated and aggressive phenotype.

Nicola McCarthy

ORIGINAL RESEARCH PAPER Snyder, E. L. et al. NKX2-1 represses a latent gastric differentiation program in lung adenocarcinoma. Mol. Cell $21 \mathrm{Mar}$ 2013 (doi:10.1016/j.molcel.2013.02.018)

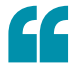

Nkx2-1 loss results in cells adopting an mRNA profile that is closest to that of the stomach epithelium g 